\title{
12N test procedure for NPSF testing and diagnosis for SRAMs
}

\begin{abstract}
Testing and diagnosis techniques play a key role in the advance of semiconductor memory technologies. The challenge of failure detection has attracted investigation on efficient testing and diagnosis algorithm for better fault coverage and diagnostic resolution. March algorithms are widely used in SRAM testing to detect and diagnose SRAM fault model since they are relatively simple and yet providing high fault coverage and diagnostic resolution. In this case to achieve high fault coverage the structure of the consecutive memory backgrounds are very important. This paper aims to prove the efficiency of March $12 \mathrm{~N}$ algorithm in term of detection and identification capability and locate the NPSF model fault. The details of test and diagnosis procedures for NPSF are demonstrated in this paper. The fault detection and diagnostic of the SRAM memories in this paper is verified and proven. The required march elements, detection requirement, detection conditions and fault syndromes are also enlightened. Furthermore, these particulars are required to determine a good algorithm other applications.
\end{abstract}

Keyword: Neighborhood pattern sensitive fault; Test procedure; March algorithm; Multi data background SRAM 